\title{
Can Music Touch the Heart? Commentary on the Benefits of Music Listening for People Living with Heart Failure
}

\author{
Renée Fleming ${ }^{1}$, Sheri L. Robb PhD, MT-BC ${ }^{2,3}$ \\ From the ${ }^{1}$ Artistic Advisor, John F. Kennedy Center for the Performing Arts; ${ }^{2}$ Professor, Indiana \\ University School of Nursing and ${ }^{3}$ Director, Indiana CTSI KL2 Young Investigators Program.
}

"Musick has charms to soothe a savage breast." William Congreve, 1697

Popular literary tradition depicts the heart as the seat of human emotion, and evidence of this is everywhere. We speak of music touching our heartstrings, we talk of having a change of heart, we place our hands over our hearts when we sing the national anthem. Of course, we know today that mental and emotional activity is centered in the brain, but can music actually affect our hearts? Is there medical and scientific evidence of music's effects on heart health?

Music Listening (ML) programs have received increased attention in the scientific literature (both experimental and interventional), and are especially attractive given their potential for broad delivery. ${ }^{1}$ In 2016, the John F. Kennedy Center for the Performing Arts and the National Institutes of Health (NIH) launched a collaborative initiative, spearheaded by one of us (RF), serving as artistic advisor to the Kennedy Center; Deborah Rutter, president of the Kennedy Center; and Dr. Francis Collins, director of the NIH. Sound Health: Music and the Mind, in association with the National Endowment for the Arts (NEA), brings together leading neuroscientists, music therapists, and arts practitioners to better understand the impact of arts on the mind and body. As a result of this initiative, in 2019, the NIH awarded \$20 million dollars in funding for music and neuroscience research over five years, signaling a growing interest in this sector.

This is the author's manuscript of the work published in final edited form as:

Fleming, R., \& Robb, S. L. (2020). Can Music Touch the Heart? Commentary on the Benefits of Music Listening for People Living with Heart Failure. Journal of Cardiac Failure, 26(7), 552-554.

https://doi.org/10.1016/j.cardfail.2020.06.013 
Findings from a new study in the current issue of the Journal suggest that listening to pre-selected classical music (30 minutes daily) at rest may help improve symptoms of anxiety and depression in people who are living with heart failure (HF), and may improve sleep quality. More specifically, patients randomized to the ML program reported significantly less anxiety and depression compared with the control group after 30,60, and 90 days of listening. Similarly, ML participants reported significantly better SF-12 mental component scores at these same time points; however, no significant differences were found for the physical component. Taken together, these findings suggest the intervention had its greatest affect in the areas of mental or emotional well-being.

Limited findings for physical well-being may have been due (at least in part) to reasonable baseline physical status inferred from New York Heart Association class, which focuses on limitations experienced during physical activity and focus on breathing, shortness of breath, and angina pain. At enrollment, $43 \%$ of participants were Class I and 50\% were Class II. In future studies, it would be interesting to look at how the intervention might affect those with more advanced NYHA class, and whether outcomes continue to be most robust in the emotional, rather than physical domains.

\section{But can we expect ML to have a spontaneous effect on physical outcomes (such as breathing and} shortness of breath), or will these changes require more intentional and volitional uses of music? As discussed by Ellis and Thayer, unlike heart rate, breathing is one of the few physiological processes that can be voluntarily controlled making it a viable target for interventions. ${ }^{2}$ Future studies that use compositional features of music to support and structure the rate of breath inhalation/exhalation, or explore the use of specific breathing exercises structured through singing and vocal exercises may prove valuable for patients with HF and may produce greater benefits on outcome measures like the SF-12 and Minnesota Living with Heart Failure Questionnaire.

We are born with inherent rhythmic capability that begins with hearing our mother's heartbeat. Humans naturally entrain to repeated rhythmic stimuli, including walking, dancing, and several other activities. Crying premature infants benefit from a music therapist's ability to sing increasingly longer 
tones, thereby calming and entraining the baby's cry. ${ }^{3}$ The act of singing can foster and improve breath control, strengthening the vocal and breathing apparatus. Listening to calming music encourages longer, fuller breathing, another example of rhythmic entrainment. In addition, singing has been shown to induce improvement in breathing in patients with chronic pulmonary disease (COPD) ${ }^{4}$

In the current study, the authors propose that relaxing and pleasant music activates parasympathetic activity while decreasing sympathetic activity. Although compositional features of music characterized as calming are known to affect various measures of stress ${ }^{5,6}$ - cultural, sociological, and individual experiences (including music-evoked memories) account for individual differences in emotional response to music that influence what may be occurring at lower brain levels. ${ }^{2}$ There is evidence that preferred music is an important factor to consider when designing ML programs, ${ }^{6}$ and that in order to evoke the strongest relaxation response, it is likely important to consider music's compositional features and preference.

But what do we mean when we talk about music preference? If the goal is to produce a relaxed state, we need to consider not only what a person likes, but also how a piece of music affects their emotional state. For example, a study that examined autonomic nervous system (ANS) reactivity to positive and negative mood induction found no direct evidence for a generalized increase in parasympathetic nervous system activity by positive mood induction tasks or parasympathetic withdrawal in response to negative mood induction tasks. ${ }^{7}$ However, participants who actually experienced the anticipated feelings of happiness in response to the positive mood induction tasks displayed an increase in heart rate variability (HRV) suggesting increased parasympathetic activity. The implication here is that pleasant, relaxing music may result in HRV changes, but the direction and strength of the result will depend on the emotional response of the listener.

To this point, it is important to acknowledge that the current study took place in Italy, participants were in their early to mid-70s, and the ML program focused on classical music alone. It is not clear how the authors measured preference, but they report that participants indicated the playlist (which included 80 
selections) met their preferences. Although this takes into account whether participants "liked" the music, less clear is whether classical music was consistent with their musical taste, listening habits, and emotional responses to the music.

In the early $20^{\text {th }}$ century, there were more limited sources for music enjoyment. Outside of live concerts, people might have generally listened to one radio station that played a single genre of music, or perhaps to a modest record collection at best. In recent decades, from the emergence of the compact disk (CD) through the rise of internet music streaming, there has been a rapid expansion of the amount and variety of music that is commercially widespread, and people have in turn become more eclectic in their musical taste. It is now more common than not for people to regularly listen to music of multiple genres and styles. There are also a multitude of relaxing examples of music present in many other genres, not just classical music. Furthermore, "common" assumptions of which music is considered relaxing also varies widely across the population. Even for one of us (RF), many conventionally "relaxing" pieces of classical music are not personally relaxing. So, as we think about how these findings may be applicable to other populations of patients with HF, it is important to consider how cultural and generational differences, along with individual experience, may affect generalization of findings.

Future studies that examine heart rate variability and emotional response may provide greater understanding about the mechanisms of action for ML in HF. ${ }^{2,6}$ In addition, individualized approaches to music selection that consider music preference, listening habits, and how individuals typically uses music to achieve a relaxed and positive emotional state (that is neither too high or too low) may produce more robust findings. Several investigators have developed music selection assessments, including individualized ML programs to promote physical activity during cardiac rehabilitation, ${ }^{8}$ reduce anxiety and depressive symptoms in nursing home residents, ${ }^{9}$ and lower anxiety in mechanically ventilated adults. ${ }^{10}$

How might social isolation affect anxiety, depression, and stress? As we think more broadly about the current intervention and implications for future work, it is important to consider demographic information 
for this population and the challenges that those living with HF encounter each day. In this study, the average age of study participants was early to mid-70s, with 34\% (about 1/3) living alone, and 52\% living with one other person. In designing additional studies, it would be interesting to know how many participants are involved in music socially - listening with others at home, attending concerts, or playing/singing in community music groups.

This brings to light the many ways music is being used by different healthcare professionals, and the potential for community-based music programs to meet the holistic needs of patients living with HF and their families. Research to advance the efficacious use of ML programs are important, because they have potential for broad delivery at a low cost. Community-based music programs, such as choral activities, are another avenue for encouraging social interaction, support, and better mental health and well-being. For example, Johnson's Community of Voices study found that, for a diverse sample of older adults, participation in community choirs led to a significant reduction in loneliness and increased interest in life. ${ }^{11}$ Finally, for patients with HF and caregivers who present with higher levels of emotional and/or physical needs, tailored interventions that incorporate ML and/or active music-making experiences along with social support may be required - such as those provided by credentialed music therapists.

How do we advance research that informs and supports the use of music for health? The NIH-Kennedy Center Sound Health Initiative is working to accelerate our understanding about the use of music for health benefit through interdisciplinary scientific collaboration. Scientific advancements in any field require clear, transparent reporting and common language that encourages cross disciplinary collaboration.

Music interventions are especially difficult to fully describe due to the complexity of music stimuli (e.g., rhythm, tempo, pitch, harmonic structure, timbre), variety of music experiences (e.g., music listening vs. active music making), and varied background/training of persons delivering interventions. The Checklist for Reporting Music-based Interventions were published to improve consistency of intervention reporting, and in turn improve validity, replicability, and clinical application of findings. ${ }^{12}$ In the current study, 
reporting strengths included discussion about the scientific premise underlying music selection, instructions for music use, playback equipment and decibel controls, dose, as well as supplemental information listing every musical selection. Unfortunately, a majority of previously published studies lack detailed information about intervention content and delivery, hindering cross-study comparisons and meaningful meta-analytic studies. ${ }^{13}$

Terminology that is well defined and consistently applied across studies is also required. A systematic review of music intervention research in healthcare highlights the importance of adopting common language, given fundamental differences in the type and complexity of interventions that are being studied, and how intervention content varies based on professional background. ${ }^{13}$ For example, use of the term "music therapy" as a categorical label for all music intervention studies is inaccurate and creates problems with cross-study comparisons, because music therapy refers to a profession (as does nursing, occupational therapy, or medicine), and as such infers delivery from a credentialed music therapist. The term "music interventions" captures the work more broadly, and includes interventions provided by the broader range of healthcare professionals and practitioners. However, neither term provides detailed information about the actual music intervention. In order to achieve even greater clarity and specificity in published reports and subsequent systematic reviews, it is important that we also use terms that clearly describe how the music was applied or experienced (e.g., singing, music listening, rhythmic auditory stimulation), while also considering the background/training of the individuals who delivered the intervention.

This is an exciting time; with the burgeoning field of integrative medicine, interest in the intersection of music and science is growing. This sector is fertile ground, with increased demand for further research from clinicians, practitioners, scientists, and the general public. Humans are - at heart - emotional, social, and creative beings who need each other, and music has the power to bridge that connection.

For more information on the Sound Health Initiative visit: 
https://www.nih.gov/research-training/medical-research-initiatives/sound-health

For more information on Music and Mind Lectures visit:

https://reneefleming.com/advocacy/music-and-the-mind/

For more information on music therapy in the United States, Europe, and the world visit:

Certification Board for Music Therapists https://www.cbmt.org/

American Music Therapy Association https://www.musictherapy.org/

European Music Therapy Confederation https://www.emtc-eu.com/

World Federation of Music Therapy https://www.wfmt.info/ 


\section{References}

1. Bradt J, Dileo C, Potvin N. Music for stress and anxiety reduction in coronary heart disease patients. Cochrane Database Syst Rev 2013(12):CD006577.

2. Ellis RJ, Thayer JF. Music and Autonomic Nervous System (Dys)function. Music Percept 2010;27(4):317-26.

3. Loewy J, Stewart K, Dassler A, Telsey A, Homel P. The Effects of Music Therapy on Vital Signs, Feeding, and Sleep in Premature Infants. Pediatrics May 2013;131(5):902-18. https://doi.org/10.1542/peds.2012-1367.

4. Canga B, Azoulay R, Raskin J, Loewy J. AIR: Advances in Respiration $\square$ Music therapy in the treatment of chronic pulmonary disease. Respiratory Medicine 2015;109(12):1532-9.

5. Bernardi L, Porta C, Sleight P. Cardiovascular, cerebrovascular, and respiratory changes induced by different types of music in musicians and non-musicians: the importance of silence. Heart 2006;92(4):445-52.

6. Tan X, Yowler CJ, Super DM, Fratianne RB. The Interplay of Preference, Familiarity and Psychophysical Properties in Defining Relaxation Music. J Music Ther 2012;49(2):150-79.

7. Kop WJ, Synowski SJ, Newell ME, Schmidt LA, Waldstein SR, Fox NA. Autonomic nervous system reactivity to positive and negative mood induction: the role of acute psychological responses and frontal electrocortical activity. Biol Psychol 2011;86(3):230-8.

8. Clark IN, Baker FA, Taylor NF. Older Adults' Music Listening Preferences to Support Physical Activity Following Cardiac Rehabilitation. J Music Ther 2016;53(4):364-97.

9. Costa FOA, Hargreaves DJ. The effect of regular listening to preferred music on pain, depression, and anxiety in older care home residents. Psychology of Music 2018;46(2):14-191. 
10. Chlan L, Heiderscheit A. A Tool for Music Preference Assessment in Critically Ill Patients Receiving Mechanical Ventila-tory Support. Music Ther Perspect 2009;27(1):42-7.

11. Johnson J.K., Stewart A.L., Acree M., Napoles A.M., Flatt J.D., Max W.B., Gregorich S.E.A Community Choir Interven-tion to Promote Well-Being Among Diverse Older Adults: Results From the Community of Voices Trial. The Journals of Gerontology: Series B, 75(3):549-559.

https://doi.org/10.1093/geronb/gby132

12. Robb SL, Burns DS, Carpenter JS. Reporting guidelines for music-based interventions. J Health Psychol 2011;16(2):342-52.

13. Robb SL, Hanson-Abromeit D, May L, et al. Reporting quality of music intervention research in healthcare: A systematic review. Complement Ther Med 2018;38:24-41. 